\title{
Post-Surgery Musical Hallucinations of a Patriotic Canadian Song
}

\author{
Camilla L. Wong, Shree Bhalerao
}

Can. J. Neurol. Sci. 2010; 37: 901-903

A 79-year-old, right-handed, widowed female with bilateral sensorineural hearing loss of a few years' duration, presented to a peripheral hospital with a fall. The fall resulted in a subcapital fracture of the right hip without head injury. She underwent a cannulated screw fixation of the right hip and was discharged to a rehabilitation facility a few days thereafter. She began to experience neck pain and progressive motor weakness of her upper and lower extremities, with sensory deficits in the upper extremities bilaterally. After a few weeks, she could only mobilize with a wheelchair. Bowel and bladder function remained intact. A magnetic resonance image confirmed a 2.4 $\mathrm{cm} \times 1.9 \mathrm{~cm}$ dural based mass seen anteriorly at the foramen magnum, causing cervicomedullary junction cord compression. She underwent an uneventful resection of the mass with a C1-C2 laminectomy (Figure). The mass was determined pathologically to be a meningioma.

Postoperatively, she reported hearing "The Maple Leaf Forever", a Canadian patriotic song, amongst some Elvis Presley favourites, though clearly had insight that these were hallucinations. In fact, the musical hallucinations began a few days following her hip repair, but she had not brought this to medical attention fearing she would be labeled as 'crazy'. The phenomenon was bilateral. There was no tinnitus. She denied any illusions, delusions, and additional perceptual disturbances.

She also had a history of depression, though stable with psychotherapy and an antidepressant. She denied any alcohol or illicit drug use. She had bilateral sensorineural hearing loss and had not worn her hearing aids since the hip surgery fearing they would be misplaced in hospital. Prior to her recent fall, she was independent for all her activities of daily living and independent activities of daily living, except for using a walker to ambulate. She was widowed, lived alone, and had two supportive daughters.

She was not experiencing low mood, but had vegetative symptoms of depression including weight loss (nine pounds since her hip surgery), fatigue, and insomnia. While dismayed at her lack of progress with physiotherapy, she remained motivated.

She was on dexamethasone as part of neurosurgical management for the meningioma. Her other medications were citalopram, amlodipine, dalteparin, alendronate, vitamin D and calcium.

Her affect was flat, but she was alert and attentive. She had sensorineural hearing loss bilaterally. She could sing the song "The Maple Leaf Forever" and return to a different verse when interrupted. Cognitive testing included a Mini Mental State Examination score of 29/30, losing a point for visuospatial construction. Other than $4 / 5$ power in the upper extremities, decreased sensation to pin prick and light touch in the upper extremities, and mildly increased tone bilaterally, the remainder of the physical examination was unremarkable.

Laboratory investigations were unremarkable. An EEG excluded temporal lobe epilepsy. A postoperative magnetic resonance image was consistent with a tumour resection without evidence of any infarct.

A psychiatry consultation determined she had a diagnosis of psychosis not otherwise specified and a major depressive disorder, recurrent-type in partial remission. She did not meet criteria for delirium nor dementia.

Dexamethasone was tapered off and citalopram was titrated up to $40 \mathrm{mg}$ once a day to target her neurovegetative symptoms of depression. Her hearing aids were repaired and worn daily. An antidepressant, rather than an antipsychotic, was chosen given the predominance of affective symptoms. Her affect improved and she progressed with physiotherapy. In spite of these interventions, she continued to experience musical hallucinations.

\section{Discussion}

Musical hallucinosis, a subtype of auditory hallucinations, though well known, is an uncommon and poorly understood phenomenon. They are characterized by a complex sound processing disorder where the perception is formed by instrumental music, sounds or songs. ${ }^{1}$

\section{Features}

The melodies are usually repetitions of well known music, and can range from religious songs, folk songs or pop songs heard during childhood or adolescence. ${ }^{2}$ An analysis of published cases on musical hallucinations revealed an association with certain clinical features, though often cases were a juxtaposition of factors. Common clinical features include acquired hearing loss, psychiatric disease, focal brain lesions, epilepsy or intoxication. The role of hemispheric dominance is contentious and the influence of musical talent remains unclear. ${ }^{1-3}$

From the Division of Geriatrics (CLW), Department of Psychiatry (SB), University of Toronto; Li Ka Shing Knowledge Institute of St. Michael's Hospital (CLW), Toronto, ON, Canada.

Received January 14, 2010. Final Revisions Submitted April 23, 2010. Correspondence to: Camilla Wong, 30 Bond Street, Toronto, Ontario, M5B 1W8, Canada. 


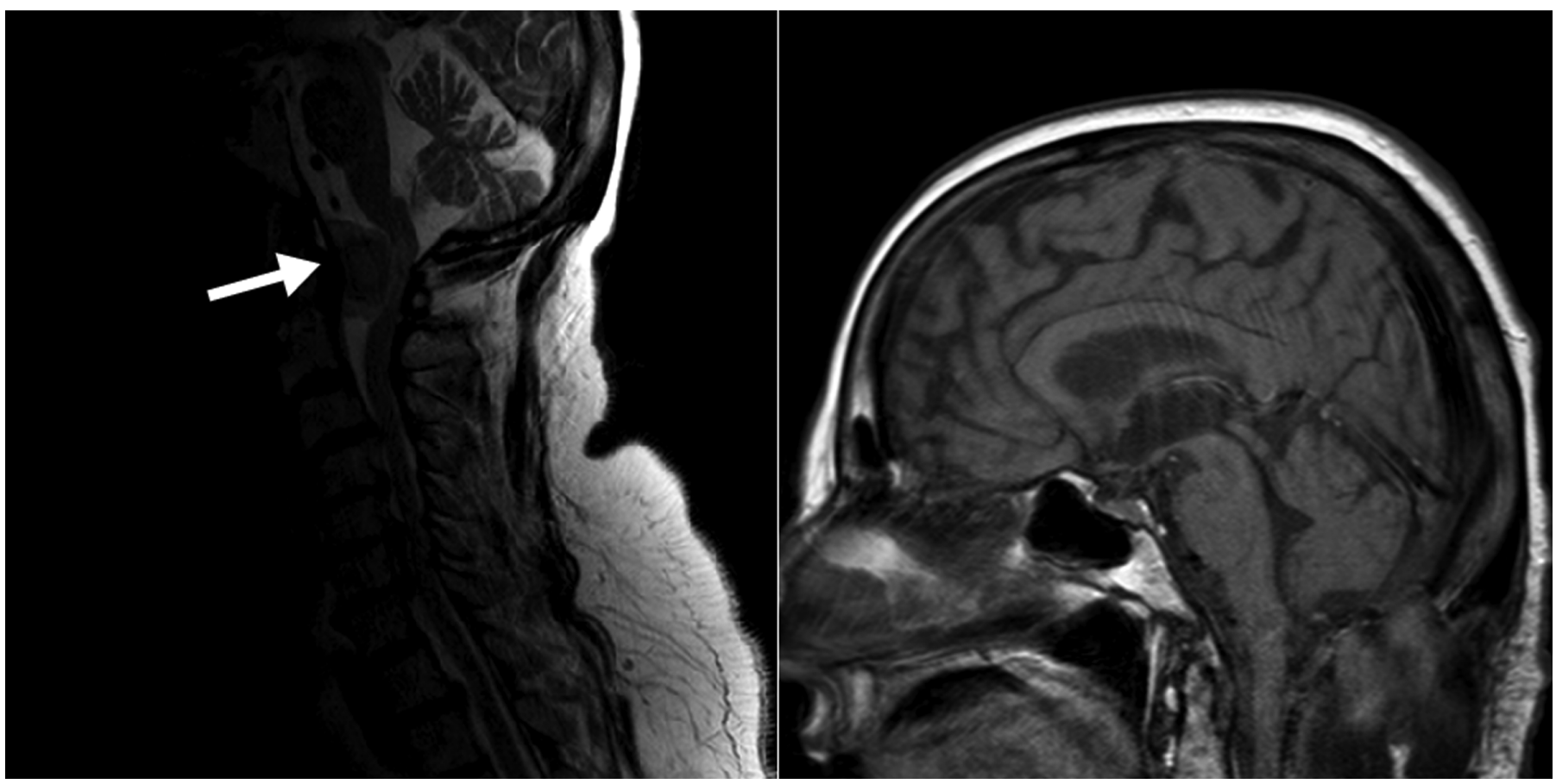

Figure: (Left) T2-weighted magnetic resonance image demonstrating a dural-based meningioma anteriorly at the foramen magnum (arrow), causing cervicomedullary junction cord compression. (Right) T1-weighted magnetic resonance image showing upper cervical cord decompression following tumour excision.

\section{Pathophysiology}

Musical hallucinations appear to be a phenomenon caused by heterogeneous mechanisms but acquired hearing loss is most often cited as an important factor. As such, it may represent an auditory form of the Charles Bonnet syndrome. ${ }^{4}$ This term usually describes formed visual perception in the absence of visual stimulation in subjects with peripheral vision loss. This invokes the concept of 'parasitic memory' or deafferentation syndrome akin to phantom limb pain in amputees; constant sensory input is usually necessary to inhibit the unwanted emergence of memory traces to consciousness. ${ }^{2}$

Structurally, the temporal lobes have been implicated and supported by studies demonstrating abnormal EEG activity of the temporal cortex ${ }^{5}$, provocation of musical hallucinations by direct stimulation of the superior temporal gyrus, and functional brain imaging. ${ }^{3}$ In a subset of cases, musical hallucinations may be focal epileptic seizures of the temporal lobe. ${ }^{2}$ Functional neuroimaging studies of music perception suggest that access to musical semantic memory involves the left middle temporal gyrus and musical semantic representations are stored in the left anterior temporal areas. ${ }^{6}$

The frequent association with psychiatric disease, generalized atrophy, or intoxication lends to the possibility that the disrupted complex neuronal network is vulnerable to external triggers. ${ }^{3}$ Interestingly, the use of benzodiazepines and tricyclic antidepressants in psychiatric disease have been reported to trigger musical hallucinations. ${ }^{5,7}$

\section{Treatment}

Most patients who experience musical hallucinations are not bothered by the symptom. Patients who are disturbed by the hallucinations may benefit from a trial of treatment of this unusual symptom. General recommendations cannot be made given the heterogeneity of the clinical spectrum. In cases of intoxication, discontinuing the causative medication may work. ${ }^{3,7}$ Ameliorating the hearing deficit or manipulating ambient sound have also been tried. ${ }^{8}$ In patients suffering from depression or schizophrenia, trials of antidepressants, neuroleptics and anticonvulsants have had variable results. ${ }^{3}$ There are case reports of success with acetylcholinesterase inhibitors. ${ }^{9}$ Successful treatment with electroconvulsive therapy has been reported. ${ }^{5}$

\section{Key Points}

The chronology of this patient's musical hallucinations followed discontinuing the use of her hearing aids. However, this case highlights that musical hallucinations usually occur in the setting of multiple risk factors. The post-operative state, depression, and a meningioma were likely contributors in this case.

- Hearing loss is neither a necessary nor usually sufficient condition for the occurrence of musical hallucinations. Other factors such as psychiatric disease, focal brain lesions, epilepsy or intoxication need to be considered. 
- Patients with musical hallucinations usually have insight that the phenomenon is not real.

- Treatment response to antidepressants, neuroleptics, anticonvulsants, and hearing aids is variable.

\section{REFERENCES}

1. Berrios GE. Musical hallucinations. A historical and clinical study. Brit J Psychiat. 1990;156:188-94.

2. Keshavan MS, Davis AS, Steingard S, Lishman WA. Musical hallucinosis: a review and synthesis. Neuropsychiatry Neuropsychol Behav Neurol. 1992;5:211-23.

3. Evers S, Ellger T. The clinical spectrum of musical hallucinations. J Neurol Sci. 2004;227(1):55-65

4. Griffiths TD. Musical hallucinosis in acquired deafness, phenomenology and brain substrate. Brain. 2000;123(Pt 10):2065-76.
5. Wengel SP, Burke WJ, Holemon D. Musical hallucinations. the sounds of silence? J Am Geriatr Soc. 1989;37(2):163-6.

6. Koelsch S, Siebel WA. Towards a neural basis of music perception. Trends Cogn Sci. 2005;9(12):578-84.

7. Fisman M. Musical hallucinations: report of two unusual cases. Can J Psychiat. 1991;36(8):609-11.

8. Fenton GW, McRae DA. Musical hallucinations in a deaf elderly woman. Brit J Psychiat. 1989;155:401-3.

9. Strauss MH, Gertz HJ. Treatment of musical hallucinations with acetylcholinesterase inhibitors. J Neurol Neurosurg Psychiatry. 2009;80(11):1298-9. 